\title{
Paediatric crossword puzzle - 6
}

Manouri P Senanayake ${ }^{1}$

Sri Lanka Journal of Child Health, 2009; 38: 106

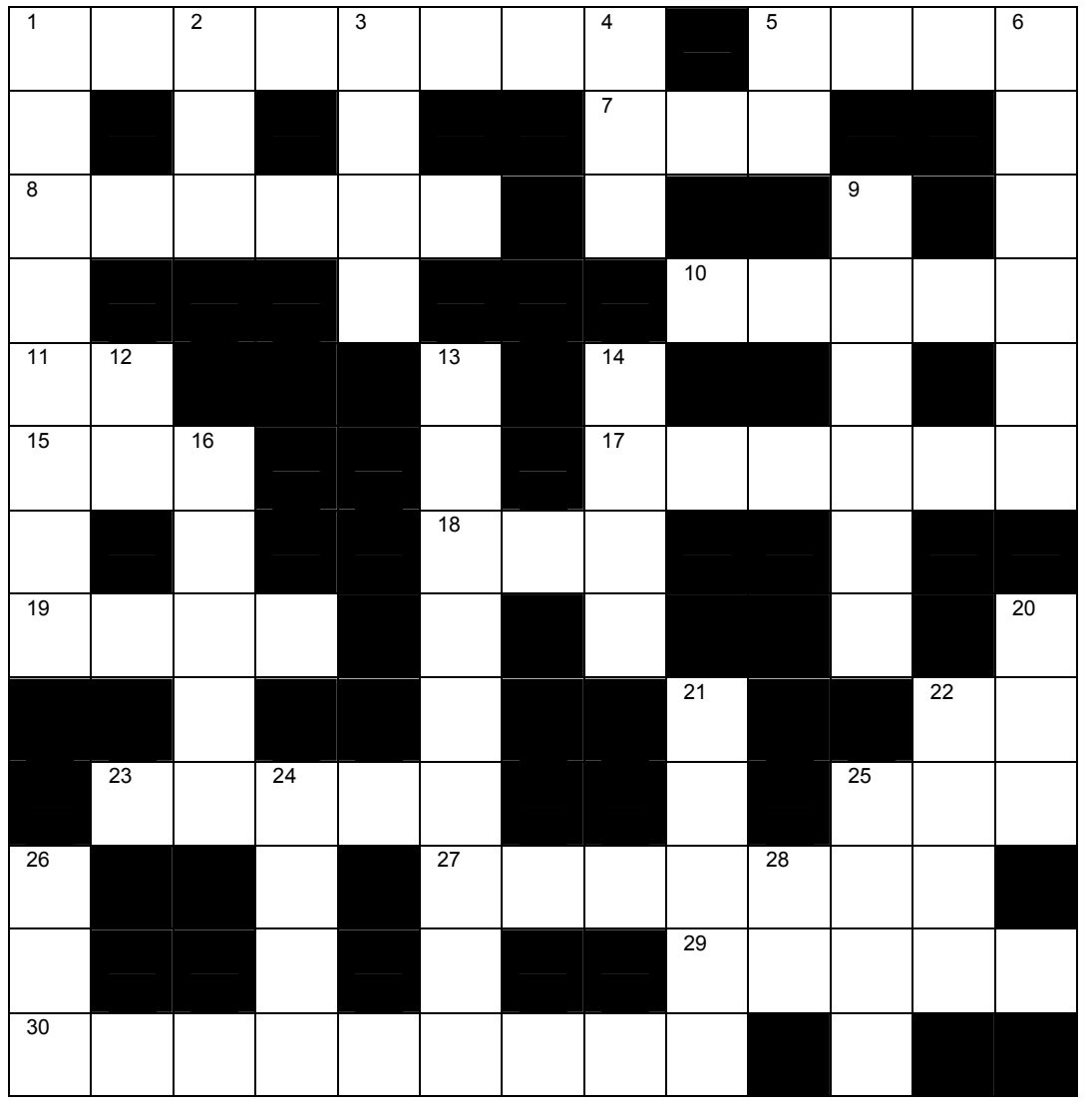

\section{$\underline{\text { Across }}$}

1. syndrome of severe protein calorie malnutrition

5. describes respiration in 'Kussmaul breathing'

7. arterio venous malformation (abbrv)

8. the base is used to alkalinize urine in renal tubular acidosis

10. an insect that transmits typhus fever

11. medication that causes pulmonary vasodilation

15. immune mediated platelet destruction (abbrv)

17. route of administration to control seizures rapidly

18. in vitro fertilization (abbrv)

19. one

23. neurodegenerative disorder caused by $\mathrm{X}$ linked lipidosis

25. cause of death (abbrv)

27. stutter

29. a small, feasibility study

30. describes diarrhoea in lactose intolerance

\section{$\underline{\text { Down }}$}

1. constipation in newborn is sometimes due to a plug of

2. principal infective reservoir of Leptospira

3. shape of thymic shadow on $\mathrm{X}$ ray

4. Widal test (abbrv)

5. endocrine disease reaching epidemic proportions in South Asia

6. endocrine gland that produces melatonin

9. the only X linked mucopolysacharroidosis

12. occupational therapist (abbrv)

13. skeletal region where rachitic process is most active

14. sign of peripheral perfusion (abbrv)

16. hairy ----- is due to genes carried on Y chromosome

20. cardiac axis in tricuspid atresia

21. glycogen storage occurs in heart

22. primitive reflex of clinical significance

24. shape of Gaussian curve

25 . cytology is the study of

26. assessed using calendar dates and skeletal maturity

28 an integral part of endocardial cushion defects

${ }^{1}$ Professor of Paediatrics, Faculty of Medicine, University of Colombo

See December 2009 issue for answers 
